\title{
A multistimulus, portable, and programmable conditioning panel for pigeons
}

\author{
LI XIA, JUAN D. DELIUS, and MARTINA SIEMANN \\ Universität Konstanz, Konstanz, Germany
}

\begin{abstract}
A portable two-response-key conditioning panel for pigeons is described. It can be attached to the subjects' home cages and allows around-the-clock experimentation. Visual stimuli are presented with light-diode display matrices. Food rewards are dispensed directly onto the stimulus/response keys with separate solenoid dispensers. Several platforms can be simultaneously controlled by a single personal computer with programs written in a simple language adapted for on-line operation. Two experiments exploring the effects of prolonged conditioning sessions upon responding and learning demonstrated the effectiveness of the system. The apparatus is an efficient and inexpensive alternative to a conventional conditioning chamber.
\end{abstract}

There is no doubt that the conditioning chamber that B. F. Skinner devised in the late 1930s is among the most enduring pieces of scientific equipment around. Known as the Skinner box, more than half a century later, it still is standard in many animal learning laboratories. As used with pigeons in experiments on the visual control of conditioned responding, it typically consists of a $40-\mathrm{cm}$ cubic sound-proofed enclosure. Translucent response keys are located on one of the vertical walls and visual stimuli are backprojected on these keys with in-line projectors. A solenoidoperated food hopper is used to offer grain rewards within a recessed bay below the keys. Mild punishment is dealt out by temporarily extinguishing the light illuminating the chamber.

While it is the case that the subjects are shielded from external interference in the box, it is also true that the environment is an unfamiliar, unnatural one, even for laboratorybred and -kept pigeons. Furthermore, the chamber is not compatible with a prolonged housing of subjects, according to current animal-welfare standards. Accordingly, the normal procedure is to remove pigeons from their habitual home cages and place them into the Skinner box for sessions lasting a few hours at most. Food deprivation has to be maintained by regular weighing and rationed feeding. The handling involved with these procedures and the strangeness of the box are stressful for pigeons. The stimulus range provided at any given time by in-line projectors is restricted. Slide projectors can be used to expand the range,

The development and research was supported by the Deutsche Forschungsgemeinschaft, Bonn. The authors thank the staff of the University workshop for technical support, Ines Krug for assistance with experiments, and Ute Delius for help with manuscript preparation. While preparing the manuscript, J.D.D. enjoyed the hospitality of J. M. Findlay and the Department of Psychology, University of Durham, England. On request, the authors will supply further technical specifications and programming information. Correspondence should be addressed to J. D. Delius, Allgemeine Psychologie, Universität Konstanz, D-78434 Konstanz, Germany (e-mail: juan,delius@uni-konstanz.de). but they are fault-prone and involve labor-intensive slide administration. Skinner boxes are expensive so that even well-financed laboratories cannot afford a large number.

Some of the drawbacks of conventional Skinner boxes and the desire to study learning processes under an at least somewhat more naturalistic condition have motivated us to develop a less contrived and less expensive conditioning device. Guided by ethological considerations, we have attempted to design a laboratory environment more similar to that in which free-ranging pigeons may normally learn during foraging. The resulting conditioning panel, ergonomic for both pigeon subjects and human experimenters, was tested in two experiments, which are briefly reported in this paper.

\section{PANEL}

The base element of the panel is a $13 \times 27 \mathrm{~cm}$ alumina plate (Figure 1). It hooks on vertically to the steel-grid home cages so that an opening $(10 \times 8 \mathrm{~cm})$ lines up with an equivalent opening in the front wall of the standard cages used in our laboratory. This is one of two side-byside identical openings that normally give the pigeons access to food and water troughs. A horizontal platform $(10 \times 6 \mathrm{~cm})$ extends outward and is level with the lower edge of the panel opening. This platform has two side-byside perforations $(2.5 \mathrm{~cm}$ diameter) with centers $5 \mathrm{~cm}$ apart. Two transparent thin Plexiglas paddles $(5 \times 3 \mathrm{~cm})$ are installed below these circular openings. These are hinged along the back edge and rest horizontally on two microswitches (Honeywell, 1SX1-T) affixed to the platform. On their upper surface, the paddles bear $0.4-\mathrm{cm}-$ high rings protruding with minor clearance up through the platforms' perforations. The response keys thus have the shape of shallow cups.

Two grain dispensers are affixed to the upper third of the base plate. Each is operated by a rotatory, springreturned solenoid (Kuhnke, D34-BOR-45, $24 \mathrm{~V} \mathrm{dc}$ ). The axle of the solenoid protrudes into the bottom end of a 


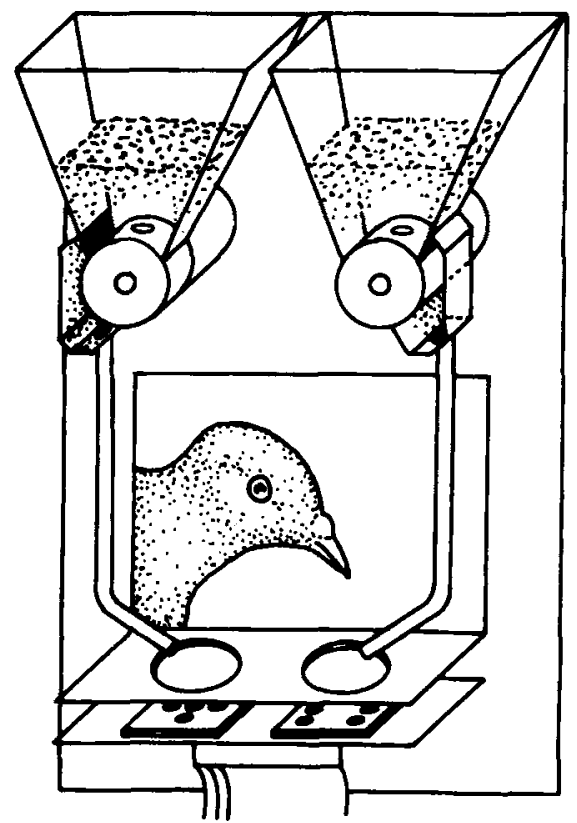

Figure 1. Layout of the conditioning panel. The panel replaces the feeding trough at the pigeon's home cage.

square-section Plexiglas funnel, which functions as a grain (millet) reservoir. The axle carries a setscrew-locked cylindrical disk ( $1 \mathrm{~cm}$ wide, $2.5 \mathrm{~cm}$ diameter) that snugly occludes (felt sealing) the bottom opening of the grain reservoir. The rim of the disk bears an elongated hemiovoid depression $(4 \times 3 \times 3.5 \mathrm{~mm})$ that takes up two to five grains of millet by gravity action when the solenoid is in the resting position. When the solenoid is activated, these grains are moved under a stiff brush fringe serving as a seal between one of the narrow walls of the reservoir and the disk (Figure 1). The $45^{\circ}$ rotation of the latter catapults the grains into a conical cavity, leading them into transparent delivery tubes. These run down along the sides of the base plate and terminate above the edges of the cupshaped keys.

A circuit board attached below and parallel to the platform carries two $5 \times 7 \mathrm{LED}$ point matrices $(1.3 \times 1.8 \mathrm{~cm}$; Knitter, LD717, green) centered below the transparent key cups. The board also carries the electronic components required to control the matrix displays (see below). A 12-pole connector establishes a 5-m multilead cable link (unscreened) between each panel and the computer interface and to external power supplies ( 5 and $24 \mathrm{~V} \mathrm{dc}$ ).

\section{CONTROL}

The control computer employed is a Commodore PC (286 Intel, 12-MHz processor), complete with keyboard, monitor, and printer. The standard configuration is supplemented with a 24 digital input/output card (Computer Board, CIO-DAS08) and an 8 optocoupler input $/ 8$ relay output card (CIO-PDIS08). The optoinputs are connected to key microswitches; the relay outputs supply grain dispensers.

The displays of up to 16 stimulus matrices can be multiplex-controlled with 10 digital interface outputs. Each two-matrix board is assigned a unique address with a DIP switch. Addresses are transmitted through lines PB0-PB7 (Figure 2). A pulse through line PC6 causes the target address to be buffered in a 74373 latch IC. A decoder consisting of two 74LS85 ICs compares this address with the board's address. The codes for the desired stimulus patterns are then transmitted through the same PB0-PB7 lines. An impulse via the PC7 line buffers these codes into two further latch ICs. These codes serve to select the corresponding LED activation patterns from each of the 2732 EPROM ICs serving the matrices. An LM555 oscillator feeds into a 74LS93 counter. The counter in turn drives the two $7445 \mathrm{BCD}$ decoders that activate the respective matrices on a cyclic row-by-row basis.

The programs controlling the operation of the panels for a given experiment are written in an expanded version of QuickBasic (Microsoft) that is analogous to the Psychobasic language described by Xia, Wynne, Münchow-Pohl, and Delius (1991) but adapted for IBM PC/MS-DOS compatibles (Xia, 1992). This new on-line control dialect, called Psyqubasic, is universally suited for psychological experiments. QuickBasic allows the incorporation of assembler routines in a library. The first part of Psyqubasic accordingly consists of a command and function library, that is a series of assembler routines supporting the input/output interface hardware and various convenient on-line operations (Pqblib.Bi). Among other things, it incorporates two input functions supporting up to 48 input channels (half of them interrupt-serviced), a counter function implementing up to 24 software counters, a timer function implementing up to 16 software timers $(0.0055$-sec precision $)$, and several output functions supporting up to 48 output channels. (Appendix A describes some of these functions.) A second part of Psyqubasic consists of an installation program that brings the system into memory residence (PsyInit). Psyqubasic can be used to run several conditioning panels, each, if necessary, with different control schedules using subroutines inserted within a main program. Tests with up to 8 panels have been run without any evidence of timing precision loss.

The visual stimuli are implemented with the above LED matrices. These stimuli can be arbitrary shapes, letters, numbers, or symbols. With a menu-driven program written in $\mathrm{C}$, even an inexperienced operator can specify any desired patterns. The computer monitor displays a $5 \times 7$ raster, and the desired stimulus is mouse-clicked into it. The codes corresponding to the pattern so specified and additional control information are stored into a file. This information is then simply transferred into the above eprom ICs with the help of an eprom programming device. Up to 256 different stimulus patterns can be stored simultaneously. The Psyqubasic function that calls up these sequentially numbered stimulus patterns on a specified display matrix is included in Appendix A. 


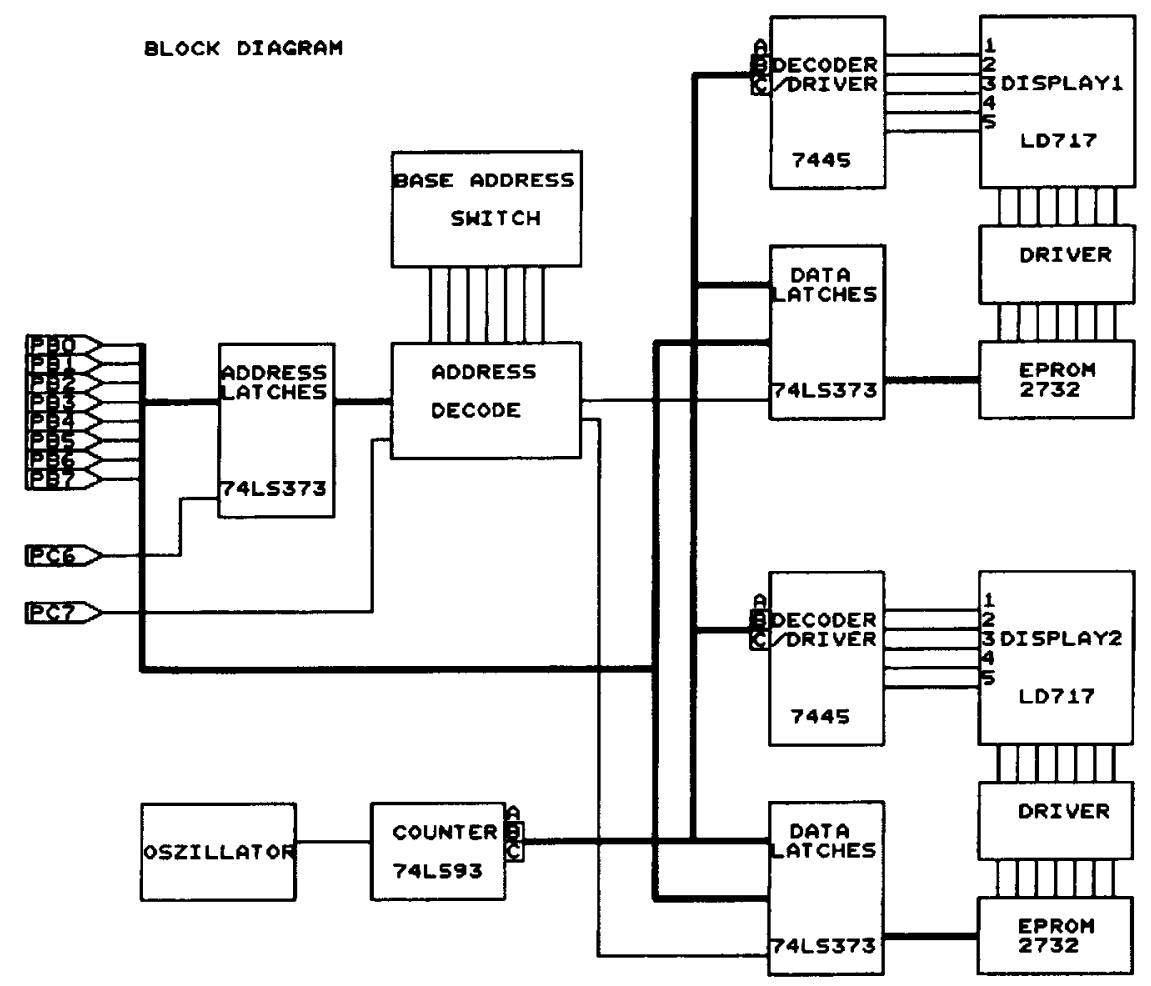

Figure 2. Block diagram of the control board with matrix stimulus displays.

\section{EXPERIMENT 1}

Experiment 1 examined the extent to which pigeons would make use of the possibility of obtaining grain reward and their performance constancy when reward is available upon an instrumental response throughout the day.

\section{Method}

Four homing pigeons (Columba livia) of local stock served as subjects. They were kept food deprived to $90 \%$ of their normal body weights through rationed once-a-day feeding. The pigeons were kept in a brightly illuminated (12:12-h light:dark) and well-ventilated animal room. The cages were stacked in standard anımal house shelves together with other cages holding pigeons kept for other purposes. Before the experiment began, the animals were autoshaped according to the procedure described by Brown and Jenkins (1968). For this, the conditioning panels were attached to the home cages for 1 day. Only one key, matrix, and feeder were in operation. A Psyqubasic program similar to that listed in Appendix B was used. A trial began with an A-shaped stimulus being displayed. If the pigeon did not peck the key three times, the stimulus extinguished after $8 \mathrm{sec}$ and reward was delivered into the key receptacle immediately afterwards. If the anımals pecked during the stimulus display, the reward was offered immediately after the third peck, and the stimulus presentation was aborted prematurely. An interval of $30 \mathrm{sec}$ with no stımulus preceded the next trial.

When the anımals pecked in at least 80 trials of a block of 100 trials (they did so within about $2 \mathrm{~h}$ ), the reward schedule was switched to a purely instrumental one. A trial began again with the presentation of the A pattern. The stimulus remained on until the pigeon pecked it three times. This led to the issue of reward with a $2-\mathrm{sec}$ feeding interval durıng which the display was extinguished. The next trial followed immediately. The experiment lasted 5 days, with the conditioning panels in place from 0830 to $1600 \mathrm{~h}$.

\section{Results}

Figure $3 \mathrm{~A}$ shows the mean daily trials completed per 15 min averaged across all 4 subjects. It is apparent that there was little day-to-day variation. During the daily $7.5 \mathrm{~h}$ each pigeon thus produced some 6,000 pecks and consumed about $20 \mathrm{~g}$ of reward millet-in this case, not needing any supplementary feeding. Although not specially recorded, observation showed that relatively few pecks were issued during the intertrial intervals. Figure $3 \mathrm{~B}$ displays the trials completed per $15 \mathrm{~min}$ plotted as a function of time of day averaged across subjects and days. It is obvious that the pigeons responded at a fairly steady rate throughout the 8 -h period, with only a minor activity peak between 1300 and $1330 \mathrm{~h}$ and a minor activity trough between 1415 and $1430 \mathrm{~h}$. Interestingly, Zeigler, Green, and Lehrer (1971) observed a minor feeding minimum at about $1000 \mathrm{~h}$ and a very definite feeding peak at about $1300 \mathrm{~h}$ in free-feeding pigeons.

\section{EXPERIMENT 2}

Experiment 2 examined the extent to which the massed training possible with the panel affected learning rates, relative to the more conventional distributed training. In a Skinner box, pigeons are usually run in daily sessions, each consisting of only $40-100$ trials and lasting between 0.5 to $2 \mathrm{~h}$. In humans, distributed practice learning is generally found to be more efficient than is massed practice learning (Underwood, Kapelak, \& Malmi, 1976), although some evidence indicates that this is not necessarily so in the con- 

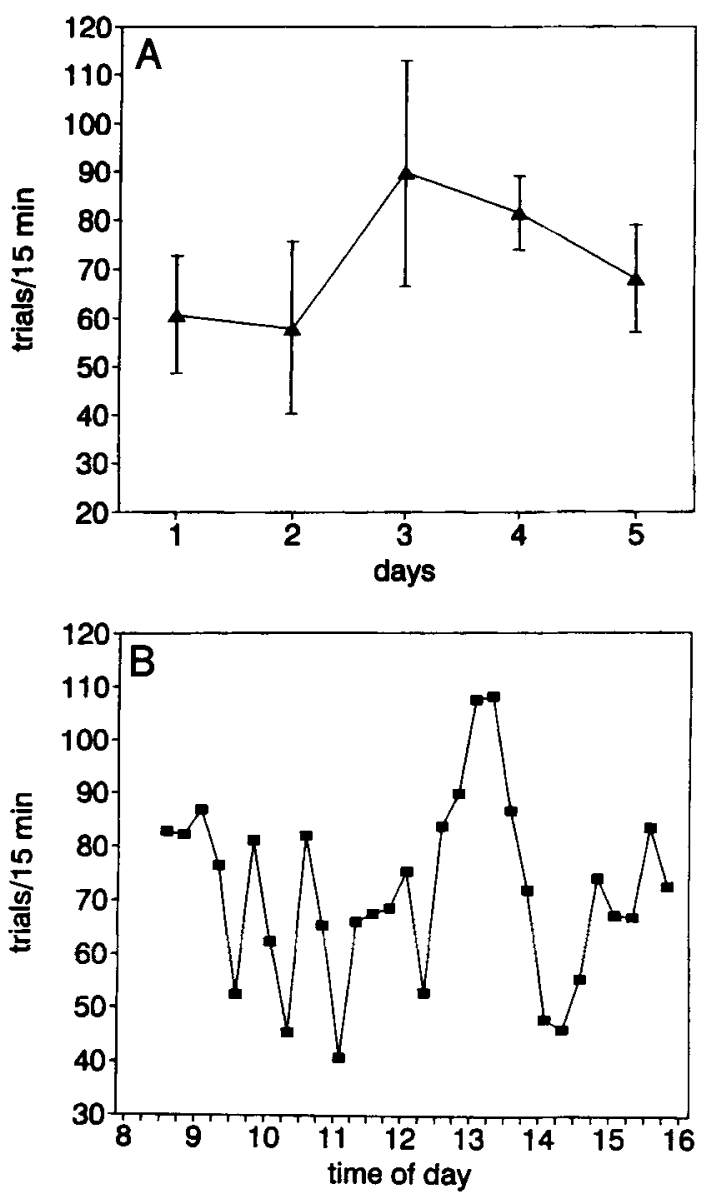

Figure 3. A: Mean daily trials completed per 15 min averaged across all 4 subjects, with standard errors. B: Trials completed per 15 min plotted as a function of time of day averaged across subjects and days.

text of motor skill learning (Lee \& Genovese, 1989). No comparable data seem to be available for pigeons.

\section{Method}

Eight new pigeons were used as subjects. As before, they were food deprived to $90 \%$ of their body weights. They were divided into two groups of 4 . One group was trained with the conditioning panels attached to their home cages. The other group was trained with an identical device attached to a separate cage but located in a cubicle away from the animal housing room. The subjects were autoshaped in these different environments as described previously except that both keys (displays, feeders) were used in a quasi-randomly alternating manner. The subjects took between 300 and 1,000 trials to achieve the $90 \%$ pecks/trial criterion; the home-cage trained group was tendentially faster.

For the discrimination training of the first group of subjects, the conditioning panels were attached to their cages in the morning and were removed only when the birds had completed 1,200 trials (about $3 \mathrm{~h}$ later). The other group of birds was trained with 12 successive daily sessions, each lasting 100 trials (about $20 \mathrm{~min}$ ), for a total of 1,200 trials. The anımals had to learn to discriminate two pairs of arbitrary-patterned stımuli, symbolized here as $\mathrm{A}+, \mathrm{B}-$, and $\mathrm{C}+, \mathrm{D}-$. A trial began with the display of one of the pairs of stimulı under the keys. Three pecks to the positive stimulus resulted in the delivery of reward on the corresponding key and a 2 -sec feeding period during which the stımuli remained visible. The next trial followed ımmed1ately afterwards. Three pecks to the negative stimulus led to a tımeout interval lasting $3 \mathrm{sec}$, in which both matrices were fully illuminated. The next trial followed immediately, and the pigeons were not allowed to correct erroneous choices. The right'left position of the stimuli was determined quasi-randomly (Gellermann, 1933). The sequencing of the two stimulus pairs was similarly quası-random. A 100 -trial retention test was run 12 days after each group had completed its training.

\section{Results}

The mean discrimination performance was assessed by calculating the percentage of correct trials. These scores are plotted as a function of the progression of training blocks in Figure 4. The retention scores are similarly shown. The massed-trials group reached a near-asymptote performance after about 400 trials, improving only slightly after that. The distributed-trials group did not evince appreciable discrimination until after 500 trials and only achieved a performance comparable to that of the former group after 1,000 trials. There was no appreciable performance difference between the groups in the delayed memory test. It does seem that pigeons are not impaired in learning and remembering visual stimulus discriminations by trial massing. Of course, the distributed-trials group was run in nonfamiliar surroundings, and this could have hampered their performance. However, this consideration also applies to conventional Skinner box training. The company of other pigeons and occasional disturbances in the animal room did not upset the performance of the massed-trials group. Less marked differences between distributed and massed training than those generally found in humans have been repeatedly reported in animals (e.g., in rats by Martasian, Smith, Nelson, Neill, \& Rieg, 1992).

\section{DISCUSSION}

The conditioning panel has proved to be an efficient method for the conditioning of pigeons. Several factors

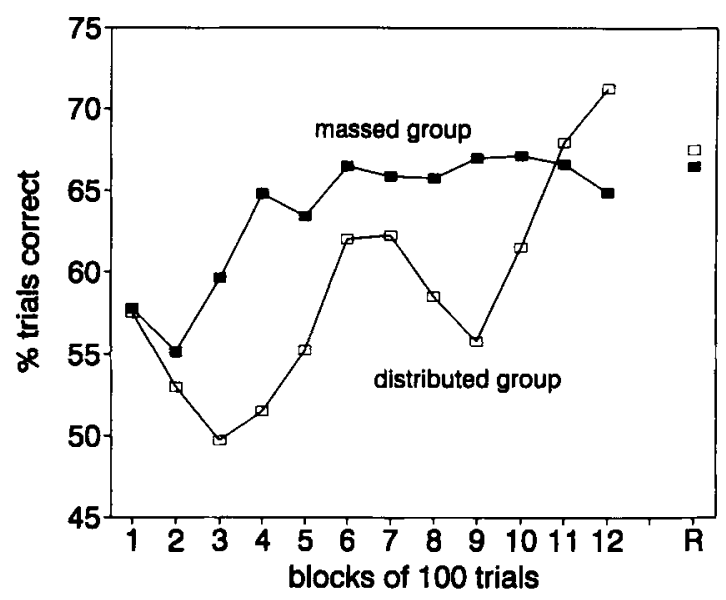

Figure 4. Mean discrimination performance of massedtrial-trained and distributed-trial-trained groups of pigeons plotted as a function of blocks of training. Each group consisted of 4 subjects. $R$, retention test. 
are likely to have contributed from the subjects' point of view. They could perform in their familiar home cage and were not exposed to stressful handling. They remained within their habitual social group in the animal room. The conditioning panel replaced their usual feeding trough. Stimuli were presented on the horizontal plane, and the responses to them were required in the same predominant plane of pigeon natural foraging activity. This arrangement has already been successfully employed by Fersen, Wynne, Delius, and Staddon (1991; see also Delius, 1992), and it corresponds with the normal viewing and the retinal specializations of pigeons (Nalbach, Wolf-Oberhollenzer, \& Remy, 1993). Rewards were delivered immediately next to the stimulus display and the response location. As soon as the panels were attached and even before the program was operational, the subjects showed marked interest in them, although they were only very mildly deprived.

The panel is also efficient from the experimenter's point of view. Continuous conditioning is possible. The massing of trials does not seem to have negative consequences. No handling of the animals is necessary to move them to the conditioning environment. It may even be possible to forgo deprivation through weighing and rationed feeding, either by offering the subjects time-limited free access to mixed grain in the evening or by replacing the millet with mixed ground grain, sieved to size and free of dust (because it tends to interfere with dispenser function) and relying on the pigeons to operantly earn all their food (closed economy conditions; Hursh, 1980). Grit, however, would need to be offered separately. If needed, larger reward portions can be easily achieved through repetitive dispenser activations.

The present conditioning panel incorporates two horizontal pecking keys. If preferred, conventional vertical keys are an option. More pecking keys present no fundamental problem. If convenient, conventional in-line projectors can replace the LED matrices as stimulus sources. Employment of miniature LC monitors (camcorder viewers; cf. Todd \& Mackintosh, 1990) could increase the range of stimuli possible. Multiple dispensers serving the same key but delivering different food qualities could be an option. Where uneaten grains remaining on the keys are troublesome, a solenoid-valve-controlled air blast or vacuum aspiration could be helpful. Where on-key grain delivery is undesirable, it is possible to use separate reward receptacles. A water dispenser, a reservoir feeding into a solenoid valve, has also been used successfully.

However, the main advantage of the conditioning panel is that it allows conditioning studies to be carried out in an environment that is closer to normal and that conforms better with modern animal-welfare standards than do the usual conditioning chambers.

\section{REFERENCES}

Brown, P. L., \& JENKINS, H. M. (1968). Auto-shaping of the pigeon's key peck. Journal of the Experimental Analysis of Behavior, 11, 1-8.

Delius, J. D. (1992). Categorical discrimination of objects and pictures by pigeons. Animal Learning \& Behavior, 20, 301-311.

Fersen, L. von, Wynne, C. D. L., Delius, J. D., \& Staddon, J. E R. (1991). Transitive inference formation in pigeons. Journal of Experimental Psychology: Animal Behavior Processes, 17, 334-341

GellermanN, L. W. (1933). Chance orders of alternating stimuli in visual discrimination experiments. Journal of Genetic Psychology, 42, 206-208.

HURSH, S. R. (1980). Economic concepts for the analysis of behavior. Journal of the Experimental Analysis of Behavior, 34, 219-238.

LEE, T. D., \& GENOVESE, E. D. (1989). Distribution of practice in motor skill acquisition: Different effects for discrete and continuous tasks. Research Quarterly of Exercise \& Sport, 60, 59-65.

Martasian, P. J., Smith, A. S., Nelson, F., Neill, S. A., \& Rieg, T. S. (1992). Retention of massed vs. distributed response-prevention treatments in rats and a revised training procedure. Psychological Reports, 70, 339-355.

Nalbach, H. O., Wolf-Oberhollenzer, F., \& Remy, M. (1993). Exploring the image. In H. P. Zeigler \& H. J. Bischof (Eds.), Vision, brain, and behavior in birds (pp. 26-46). Cambridge, MA: MIT Press.

TODD, I. A., \& MACKINTOSH, N. J. (1990). Evidence for perceptual learning in pigeons' recognition memory for pictures. Quarterly Journal of Experimental Psychology, 42B, 385-400.

UNDERWOOD, B. J., KAPELAK, S. M., \& MALMI, R. A. (1976). The spacing effect: Additions to the theoretical and empirical puzzles. Memory \& Cognition, 4, 391-400.

XIA, L. (1992). Psychobasic, eine Basic Enweiterung mit entsprechender Hardware für die Ansteuerung und Datenerfassung in psychologischen Experimenten [Psychobasic: A Basic extension with corresponding hardware for the control and evaluation of psychological experiments]. Diploma thesis, Technical College of Konstanz, Konstanz, Germany.

Xia, L., Wynne, C. D. L., MÜnChow-Pohl, F. von, \& Delius, J. D. (1991). PSYCHOBASIC: A BASIC dialect for the control of psychological experiments with the Commodore-64 and DELA interfacing. Behavior Research Methods, Instruments, \& Computers, 23, $72-76$.

Zeigler, H. P., Green, H. L., \& Lehrer, R. (1971). Patterns of feeding behavior in pigeons. Journal of Comparative \& Physiological Psychology, 76, 468-477.

APPENDIX A

\section{Some of the Special Psyqubasic Commands and Functions}

Muster displaynumber, patternnumber (on)

Muster displaynumber, 0 (off)

SetRelay, relaynumber, 1 (= on)

SetRelay, relaynumber, 0 (= off)

SetTimeEv timernumber, timeinterval, eventnumber

Time.Ttimernumber

SetIsoCtrnEv inputnumber, countnumber, eventnumber

Ctrn.Ccounternumber

Gellermann\% (variable \%)
Displays (extinguishes) specified stimulus pattern on specified display matrix

Closes (opens) specified interface relay (here, grain dispensers)

Specified event is initiated after specified time (in seconds) has elapsed on specified timer

Retains eventnumber specified in above statement

Specified event is initiated after pulses (here, keypecks) on specified optoinput has reached specified count

Retains eventnumber specified in above statement

Yields a 0 or a 1 according to a quasi-random sequence

Note--The dialect also recurs to the full complement of QuickBasic commands and functions. 


\section{APPENDIX B \\ Example of a Psyqubasic Program}

This program implements the autoshaping procedure described in the text for Experiment 2. For brevity, this listing includes only the essentials and is reduced to control only one platform, instead of four. For legibility, Psyqubasic peculiarities are in bold print and remarks are in italics.

REM SINCLUDE: 'Pqblib.Bi': REM incorporates Psyqubasic commands and functions

DIM PsyEv AS PsyEventType

PsyInit: REM activates and initializes Psyqubasic

SetRelay 0, 0: SetRelay 1, 0: Muster 0, 0: Muster 1, 0: REM sets outputs to zero

trialn $\%=0:$ REM sets trial counter to zero

SetTimeEv 0, 1, 100: REM zeroth timer, start delay, go to stimuli

WHILE INKEYS $<>$ CHRS(Esc) AND trialn $\%<100$

PsyEvent PsyEv: REM definition

IF PsyEv.Time.T0 = 100 THEN: REM a jump from beginning or intertrial

IF Gellermann \%(trialn\%) =1 THEN: REM quasirandom choice of right display/key

Muster 1, 1: REM right display, stimulus on

SetIsoIPCtrnEv 1, 3, 200: REM right key, 3 pecks, go to reward

state\$ = "stimulus right": PRINT state\$

ELSE: REM quasirandom choice of left display/key

Muster 0, 1: REM left display, stimulus on

SetIsoIPCtrnEv 0, 3, 200: REM left key, 3 pecks, go to reward

state $\$=$ "stimulus left": PRINT state\$

END IF

SetTimeEv 0, 8, 200: REM zeroth timer, max. stimulus duration, go to reward

END IF

IF PsyEv.Ctrn.C0 = 200 THEN: REM a jump from left key after 3-peck completion

SetTimeEv 0, 2, 300; REM zeroth timer, dispenser time, go to interval

SetRelay 0, 1: REM left dispenser, on

Muster 0, 0: REM left display, stimulus off

responsesleft $\%=$ responsesleft $\%+1:$ REM count of responses on left key

state\$ = "reward left": PRINT state\$

END IF

IF PsyEv.Ctrn.C1 = 200 THEN: REM a jump from right key after 3-peck completion

SetTimeEv 0, 2, 300: REM zeroth timer, dispenser time, go to interval

SetRelay 1, 1: REM right dispenser, on

Muster 1, 0: REM right display, stimulus off

responsesright $\%=$ responsesright $\%+1:$ REM count of responses on right key

state $\$=$ "reward right": PRINT state\$

END IF

IF PsyEv.Time.T0 = 100 THEN: REM a jump from max. stimulus duration when no 3-peck completion

IF Gellermann $\%$ (trialn $\%)=1$ THEN: REM right stimulus was on

SetRelay 1, 1: REM right dispenser, on

Muster 1, 0: REM right display, stimulus off

state\$ = "reward right": PRINT state\$

ELSE: REM left stimulus was on

SetRelay 0, 1: REM left dispenser, on

Muster 0, 0: REM left display, stimulus off

state $\$$ = "reward left": PRINT state\$

END IF

SetIsoIPCtrnEv 0, 0, 0: REM disables left key counter

SetIsoIPCtrnEv 1, 0, 0: REM disables right key counter

SetTimeEv 0, 2, 300: REM 0th timer, dispenser time, go to interval

END IF

IF PsyEv.Time.T0 = 300 THEN: REM a jump from reward

SetRelay 0, 0: REM left dispenser, off

SetRelay 1, 0: REM right dispenser, off

trialn $\%=$ trialn $\%+1: R E M$ trial count increment

state\$ = "interval": PRINT state\$

SetTimeEv 0, 30, 100: REM zeroth timer, trial interval, go to stimuli

END IF

WEND

PsyEnd: REM deactivates Psyqubasic and returns to QuickBasic

END

(Manuscript received September 12, 1994;

revision accepted for publication February 8, 1995.) 